\title{
A Proof of Goldbach's binary conjecture
}

\author{
Author : Sitangsu Maitra (email : maitra.sitangsu@gmail.com)
}

Abstract : The proof includes a column of similar steps and its aspects, treated in a special way. The concept of Unique Path of primes is explained and its effects are shown. In the midway of the proof, it is postponed for a while and a deviation from the course is taken to introduce a probably new axiom. Afterwards the proof restarts again, then definitions of 'list' \& 'choice' are given and a claim is proved regarding them, two lemmas and their effects on the choice of a recycled prime discussed, hence using the axiom and other results the conjecture is proved.

Please note : Every symbol ' $p$ ' with or without any suffix denotes some prime number. a|b means $\mathrm{a}$ divides $\mathrm{b}$ and $\mathrm{a}{ }^{\circledR} \mathrm{b}$ means a doesn't divide $\mathrm{b}$. $\mathrm{n}$ is a natural number. The word 'prime' will hereafter mean prime number and 'even' mean even positive integer. The sign ' $\exists$ ' means 'there exist'.

First stage : There is at least one prime $p(3 \leq p<n)$ for every $2 n>6$ such that $p \circledR 2 n$.

Proof: For any even $2 n>6$, at least one of the evens $2 n-2$ and $2 n+2$ is not an integral power of 2 . Now $n-1$ or $n+1$ is divisible by at least one prime $\mathrm{p}(3 \leq \mathrm{p}<\mathrm{n})$.

So $p \mid 2(n-1) \Rightarrow p \circledR 2(n-1)+2 \Rightarrow p \circledR 2 n$, or alternatively $p \mid 2(n+1) \Rightarrow p \circledR 2(n+1)-2 \Rightarrow p \circledR 2 n$

Suitably using any of the above two alternative results we can prove the claim.

Second stage : Let $2 n>6$. Now concider a prime $p_{1}\left(3 \leq p_{1}<n\right)$ such that $p_{1}{ }^{\circledR} 2 n$. Now let $2 n-p_{1}$ is divisible by a prime $\mathrm{p}_{2}$, where $\mathrm{p}_{2}<\mathrm{n}$.

So there can be a column of similar steps :

$\exists p_{2}$, such that $p_{2} \mid 2 n-p_{1}$, where $p_{2}<n$

$\exists \mathrm{p}_{3}$, such that $\mathrm{p}_{3} \mid 2 \mathrm{n}-\mathrm{p}_{2}$, where $\mathrm{p}_{3}<\mathrm{n}$

$$
\begin{array}{lll}
\ldots & \ldots & \ldots \\
\ldots & \ldots & \ldots \exists \mathrm{p}_{\mathrm{k}}, \text { such that } \mathrm{p}_{\mathrm{k}} \mid 2 \mathrm{n}-\mathrm{p}_{\mathrm{k}-1}, \text { where } \mathrm{p}_{\mathrm{k}}<\mathrm{n}
\end{array}
$$

( Since any $2 \mathrm{n}-\mathrm{p}_{\mathrm{k}-1}$ is smaller than $2 \mathrm{n}$, it is clear that until $2 \mathrm{n}-\mathrm{p}_{\mathrm{k}-1}$ remains composite, $\mathrm{p}_{\mathrm{k}}$ is necessarily smaller than $\mathrm{n}$ and evidently odd.)

The primes $\mathrm{p}_{2}, \mathrm{p}_{3}, \ldots$ are taken in such a manner, as far as possible, that each one is different from all the other primes (including $\mathrm{p}_{1}$ ) appearing previous to itself in the above steps.

[Clarification : $2 \mathrm{n}-\mathrm{p}_{1}$ is odd and has no prime factor which can be equal to $\mathrm{p}_{1}$, otherwise $\mathrm{p}_{1} \mid 2 \mathrm{n}$.

Now the above manner of selections of prime factors works at least for $\mathrm{p}_{1}$ and $\mathrm{p}_{2}$. Therefore we can reasonably presume that there is an arbitrary limit upto how far this manner of selections of primes works.]

It can easily be proved that no such prime divides $2 \mathrm{n}$ ( since any $\mathrm{p}_{\mathrm{k}} \neq \mathrm{p}_{\mathrm{k}-1}$ ).

The operation of getting $\mathrm{p}_{2}, \mathrm{p}_{3}, \ldots$ must end at some $\mathrm{p}_{\mathrm{k}}$, otherwise there will be infinite number of different primes $<\mathrm{n}$; $\mathrm{k}$ is a finite positive integer. We henceforth shall call $\mathrm{p}_{1}$ as 'starting prime'. We further call $\mathrm{p}_{2}, \mathrm{p}_{3}, \ldots, \mathrm{p}_{\mathrm{k}}$ (all being different, where $\mathrm{p}_{\mathrm{k}}$ is 'one' last available of them; allowing 
provision for the possibilities of having, more than one, eligible primes to the purpose of finding one such last available, from the same step, so as not to lose any kind of generality, that is why 'one' is used instead of 'the'; however this is unlikely to disturb the the following reasonings) as different outputs or simply as outputs.

[Note : The number of primes appearing in such a column of steps can't be smaller than 2, since only one prime on its own can't make any such column or step.

And take the example : $3 \circledR 2 \times 14$ so, $\exists 5$ such that, $5 \mid(2 \times 14-3)$ and $2 \times 14-5$ isn't composite and in this case $3<5<14<23<2 \times 14$. Therefore the column of steps ends here (at the step giving out its own last output), is comprised of only one step having just two elements, i.e, the starting prime and the last output. Therefore $\mathrm{k}=2$ in this case.

So we can conclude, for any such column $\mathrm{k} \geq 2$.]

Let the course of the proof be postponed for a while to discuss a topic. It is a common sense that we can omit anything from a written or mentioned expression or statement. For that purpose we simply need to wipe out or erase the purported object from the expression or the statement. But when the question comes to the dealing with its logical aspect, we need to introduce an axiom. Namely $\bullet \bullet$

Axiom of omission : We can omit or erase anything from an expression/statement or a system of expressions and/or statements, everything being viewed in some context (i.e, the concepts, logical setup, reasonings etc, all whatever logically and conceptually pertain to the expression/statement or system of expressions and/or statements), if and only if, the rest of it bears some logical meaning in that same context.

The axiom is just a logical interpretation of certain human discretion taken in common sense perspectives. Whatever be its technical interpretation, the axiom always determines the viability of any omission viewed in a context.

For our purposes in the following discussions we shall quite justifiably interpret the 'omission of something from something' as the 'imagination of no existence of former in anywhere of the later', judging only from, whatever would be obvious from the mentioned things there.

Return to the proof

Observation (1) : For a particular $\mathrm{p}_{1}$ we can choose arbitrarily particular $\mathrm{p}_{2}, \mathrm{p}_{3}, \ldots, \mathrm{p}_{\mathrm{k}}$ ( $\mathrm{p}_{\mathrm{k}}$ being the last available different output for the column of steps mentioned before, where $\mathrm{p}_{1}$ is the starting prime) and in this way they constitute a Unique Path : an ordered list of successive particular selections (one time particular selections placed in a particular order, those which can be attributed to this list within the scopes available) from the prime factors of various $2 n-p_{t}$ 's, $p_{t}$ 's starting from $\mathrm{p}_{1}$, where $\mathrm{p}_{1}$ is also included in the same list and put in the first place. Such Unique Path is always strictly ordered along the column of steps from which it derives.

Observation (2): $\mathrm{p}_{\mathrm{k}}$ being the last different output $(<\mathrm{n})\left\{\mathrm{p}_{\mathrm{k}}\right.$ available from $2 \mathrm{n}-\mathrm{p}_{\mathrm{k}-1}$ as a factor of it $\}$ in

the said column of steps, proceeding similarly beyond it we get $p_{k+1}$ from $2 n-p_{k}$, where $p_{k+1} \mid 2 n-$ $\mathrm{p}_{\mathrm{k}}$ with the exception that in this case $\mathrm{p}_{\mathrm{k}+1}$ not necessarily $<\mathrm{n}$. Besides, as this step goes past that of the last different output, the stipulation for outputs to be different from the previously appeared primes in the Unique Path, vanishes automatically for $\mathrm{p}_{\mathrm{k}+1}$. 
Now $\mathrm{p}_{\mathrm{k}+1}<\mathrm{n}$ implies $\mathrm{p}_{\mathrm{k}+1}$ is a recycled prime, i.e, $\mathrm{p}_{\mathrm{k}+1}$ is one of $\mathrm{p}_{1}, \mathrm{p}_{2}, \mathrm{p}_{3}, \ldots, \mathrm{p}_{\mathrm{k}}$ (since $\mathrm{p}_{\mathrm{k}}$ is the last available different output for that said column of $\mathrm{k}-1$ unique steps, $\mathrm{p}_{1}$ being the starting prime).

We define 'a list' as a successive mentioning of items (ignore the commas) and 'a choice' as a selection of mentioned item/s. Evidently a list is an expression. We propose that, we choose only one item from a particular list $\Rightarrow$ we omit the rest of the items from the list (provided the list contains more than one items as it's elements) $\cdots$ (Lemma on list) Proof : If not so.

Since a list is an expression of mentioned items and we have to choose from the list, if we retain at least another item in the list, other than the one intended for this choice,

there will be at least two mentioned items for a choice, where none can be excluded. But we have to choose (i.e, select the mentioned) only one item as per requirement. So there is a contradiction.

Hence our claim is true.

Let $\mathrm{p}_{\mathrm{k}+1}$ is a recycled prime. Therefore it can be taken from either case (1) the set of all elements of the Unique Path, and there is no unique order for the elements of a set, which implies more than one lists, of different particular orders for their respective elements (where number of elements is $\geq 2$ ), can be made with the elements (all and nothing more for every such list) of the set $\cdots$

[Note: When we discuss anywhere in this proof, about a list formed from a set or with the elements of a set, we should keep in mind that there is no element in that list, which is not an element of the same set]

We will now prove two important lemmas.

Lemma (a) : We can choose an element from a non empty set $\Rightarrow$ we can choose it from a list that can be formed with the elements (not necessarily all) of the same set, where one of them is the element in question.

Proof : If we can choose an element from a non empty set then the element is a member of the set.

So the list described in the above proposition can be formed from the mentioned non empty set there $\Rightarrow$ the element in question is mentioned somewhere in the list and therefore we can undoubtedly choose it from that list (as we have already admitted that we select it from the set, therefore it is already selected and it is mentioned in the list too, therefore the result follows.)

This proves the lemma (a).

Now the another lemma.

Lemma (b) : If an element $x$ can be chosen from a non empty set $S$ such that $x \in S$, then the operation of choosing it from the set $\mathrm{S}$, can only be performed in the way of choosing it from the list that can be formed with the elements of $S$ (not necessarily consisting of all elements of $S$ ), $x$ being one of those elements.

Proof: Since S is non empty,

we choose an arbitrary element of $\mathrm{S}$ and examine if it is $\mathrm{x}$. If yes, we stop the operation of choosing (Note : a single element too can make a list). If it is not $\mathrm{x}$, we have to put it aside and

choose another element from the subset $S_{1}$ of the set $S$, where $S_{1}$ contains every element of $S$ except the element chosen first and put aside, examine it as before and so on. The successive operations continue until we get $\mathrm{x}$. (Note : It is the only way to choose $\mathrm{x}$ from $\mathrm{S}$ ) 
Now in this process we get a list as $\mathrm{x}_{1}, \mathrm{x}_{2}, \ldots, \mathrm{x}$, from which evidently we get $\mathrm{x}$ \{ since the operations have to be successive and also by force of lemma (a)\}

This proves the lemma (b).

(Note : If we get $\mathrm{x}$ before finishing with all the elements of $\mathrm{S}$, then we

can continue collecting rest of the elements of $\mathrm{S}$ as being not $\mathrm{x}$ and put them in succession after $\mathrm{x}$, so that we can make a list covering every element of $\mathrm{S}$, from which $\mathrm{x}$ can be chosen)

Now the above lemmas lead to a more specific option for choosing the recycled prime, so to say that $\{$ continuing from case (1) $\} \cdots \cdot$, or case (2) it can be taken from the Unique Path only (as a method of choice of the recycled prime in question), where the existence of every element (other than the first) of this Unique Path $\Leftrightarrow$ the existence of corresponding step in the column from which the Unique Path derives.

Explanation: Take the case (1).

Choosing $\mathrm{p}_{\mathrm{k}+1}$ from the set of all elements of the Unique Path implies choosing it from at least one list having the elements of the set (all and nothing more, since a list that can be formed with the elements of a proper subset of the original set mentioned above is unacceptable in the case of such recycling purpose, see next Note). [From lemma (a) \& (b)]

If the recycled prime only to be taken from such a list described above, having an order (a list always has some particular order for its elements) different from that of the Unique Path, i.e, relative positions of other elements of the list w.r.t the recycled prime to be chosen as $\mathrm{p}_{\mathrm{k}+1}$, are different from those of the Unique Path,

then either the other list (which is not the Unique Path) cannot be derived by any column of steps such as mentioned before, or (if it at all could be derived so) the list is a different Unique Path made with the same elements (all and nothing more) of that original one (any such Unique Path remains unique only w.r.t one time particular selection of primes placed in a particular order, those which can be attributed to that Unique Path within available scopes).

Both the possibilities contradict the hypothetical situation of existence of the said column of steps or related uniqueness of the original Unique Path. [Note : For similar reason a list formed from a proper subset of the set mentioned in case (1) is unacceptable here ('unacceptable' doesn't mean impossible, it renders as just unacceptable for a certain purpose only,i.e, for the purpose of choosing a recycled prime as in this case; besides, it is unnecessary too, since choosing an element from a subset does always imply choosing it from the concerned superset too)]

Again, since each step in such a column corresponds to an unique element (except the first) of the associated Unique Path and conversely (as obvious from the column),

therefore the existence of every element (other than the first) of the Unique Path $\Leftrightarrow$ the existence of corresponding step in the column from which the Unique Path derives.

Summing up we can say, the case (2) is more definite and the only acceptable one (as a method of choice for selection of a recycled prime) as compared to other possibilities discussed above, for our purpose in this context (i.e, when $\mathrm{p}_{\mathrm{k}+1}$ is a recycled prime).

[Clarification : Here the words ' more definite ' don't negate the condition of choosing the recycled prime from the set of all elements of the Unique Path, but actually retain it, and ' other possibilities discussed above ' mean the possibility of choosing the recycled prime from any list other than the original Unique Path. In fact, choosing the recycled prime from the set of all elements of the original Unique Path $\Leftrightarrow$ choosing it from that Unique Path.]

Further, in this context we claim : the omission of an output from an Unique Path $\Rightarrow$ the omission of corresponding generating step from the column from which the Unique Path derives. $\cdots \cdots(3)$

Proof : let the proposition is not true. 
Then despite the omission of an output from the Unique Path, there exists corresponding generating step of it in the column mentioned above (in at least one case).

But the existence of the above step in the column $\Rightarrow$ the existence of the output in question in the Unique Path \{from the case (2) above\}, which contradicts the hypothesis that the output is omitted from the Unique Path.

Therefore our claim is true.

We can establish a similar result for the starting prime too. That is,

omission of the starting prime from a Unique Path $\Rightarrow$ omission of the

first step of the column from the column itself, i.e, the column from which the Unique Path derives. $\cdots \cdots(4)$

Proof: Let the above proposition is not true.

Then despite the omission of the starting prime from the Unique Path, there is existence of the first step in the column mentioned in the above proposition (in at least one case). That first step in due course gives rise to the same column of steps again and thereby its own Unique Path.

But this Unique Path is the same one mentioned in the above proposition (since the column and the Unique Path, both are unique for each other), which contains the same starting prime as its element. This contradicts the hypothesis that the starting prime is omitted from the Unique Path.

Therefore the above claim is also true.

Since $\mathrm{p}_{\mathrm{k}+1}$ is a singularly mentioned prime (i.e, only one value can be attributed to it from arbitrary options), a recycled prime taken as $\mathrm{p}_{\mathrm{k}+1}$ is also a singularly mentioned identity (i.e, only one prime that can be arbitrarily opted for that purpose), which implies we have to choose only one prime from that particular list of different primes (i.e, the Unique Path).

Now for an arbitrarily particular $\mathrm{p}_{1}$ we obtain a Unique Path of successive particular selection of primes as described in Observation (1)\}, which must contain a unique starting prime and a unique last output w.r.t the path itself (otherwise we will deny that it is a Unique Path).

Since a Unique Path itself is an ordered list,

omission of any of these two from this Unique Path (i.e, the imagination that there is no existence of any one of these two in anywhere of this Unique Path) doesn't make any logical meaning for the residual expression (i.e, the residual list) containing any of the rest (i.e, those except $\mathrm{p}_{1}$ or $\mathrm{p}_{\mathrm{k}}$ or both of them as the case of corresponding omissions may be) of its elements (not necessarily all of them), in the process regarding the choice of a recycled prime as was stated earlier, where existence of the Unique Path and thereby the column of steps from which it derives, should be taken as presuppositions (i.e, as necessary conditions) for the choice of a

recycled prime to appear as $\mathrm{p}_{\mathrm{k}+1}$.

[Clarification: The above omissions implies the Unique Path is devoid of a starting prime and/or a last output (not necessarily they are the only primes to be considered in question of omission, there can be possibilities for other outputs except them as well for omission, though necessarily alongwith any of the first two specified above in every such case) $\Rightarrow$ the aforementioned column is devoid of corresponding generating step of the last output \{from claim (3)\}, i.e, the step which is the unique end of this column having a finite number of steps, or, the very first step ffrom claim (4) \} which is unique and unavoidable for any such column of steps, (alongwith probable omissions of other steps, if so happens, those don't fall in these two category specified here), and thus the whatever (allowing provisions for the possibilities of the omissions of outputs different from $\mathrm{p}_{1}$ and $\mathrm{p}_{\mathrm{k}}$, so as not to lose any kind of generality) such residual part of the Unique Path in the context of whatever logical set up and conclusion that have been established regarding the 
column and the concerning Unique Path, upto this point, goes undefined, since no such column of steps can be formed except those essential two components (the first and last steps) for any value of $\mathrm{k} \geq 2$.]

Therefore by Axiom of omission, any of the above two omissions, i.e, $\mathrm{p}_{1}$ or $\mathrm{p}_{\mathrm{k}}$, from the Unique Path in the context described in the clarification above, is impossible,

which means after every possible valid omission from the above mentioned ordered list (i.e,

the Unique Path) there remains at least two primes to choose as $\mathrm{p}_{\mathrm{k}+1}$, none of which is omittable.

So we can say that we can't omit, 'all primes other than that intended one for recycling purpose', from the Unique Path and as the very choice from this ordered list, in question, requires at least one of the primes $\mathrm{p}_{1}$ or $\mathrm{p}_{\mathrm{k}}$ be removed from it besides all other required omissions (see lemma on list),

and since the recycled prime in question must be chosen from that Unique Path only from case (2) discussed before\},

it implies we can't choose only one recycled prime (therefore any recycled prime at all, in fact) as $\mathrm{p}_{\mathrm{k}+1}$ from that Unique Path.

Summing up the above discussions

we conclude that $\mathrm{p}_{\mathrm{k}+1}$ can't be recycled, that implies $\mathrm{p}_{\mathrm{k}+1}$ isn't $<\mathrm{n}$, and since $\mathrm{p}_{\mathrm{k}}<\mathrm{n}$,

we are bound to accept the conclusion that $\mathrm{p}_{\mathrm{k}+1}>\mathrm{n} \Rightarrow \mathrm{p}_{\mathrm{k}+1}=2 \mathrm{n}-\mathrm{p}_{\mathrm{k}}$.

$\left[2 n-p_{k}\right.$ can't have a factor that is grater than $n$ and smaller than itself, and $p_{k+1} \neq n$ for obvious reasons.]

Therefore, $2 \mathrm{n}=\mathrm{p}_{\mathrm{k}}+\mathrm{p}_{\mathrm{k}+1}$

Take a look back at the beginning of the Second stage $\cdot . \bullet$

Contrary to what we have assumed at there, if $\mathrm{p}_{2}$ isn't $<\mathrm{n}$, then as $\mathrm{p}_{1}<\mathrm{n}, \mathrm{p}_{2}$ becomes $>\mathrm{n}$. This implies $2 \mathrm{n}=\mathrm{p}_{1}+\mathrm{p}_{2}$

[ Reasons are similar as above]

Finally, over the question whether the integers $6 \& 4$ comply to Goldbach's strong conjecture, we write $6=3+3$ and $4=2+2$

Therefore Goldbach's strong conjecture holds for every $2 n \geq 4$. 